University of Nebraska - Lincoln

DigitalCommons@University of Nebraska - Lincoln

Faculty Publications, Department of Psychology

Psychology, Department of

May 2006

\title{
Rats' novel object interaction as a measure of environmental familiarity
}

Jamie L. Wilkinson

University of Nebraska-Lincoln

Laura Herrman

University of Nebraska-Lincoln

Matthew I. Palmatier

University of Nebraska-Lincoln

Rick A. Bevins

University of Nebraska-Lincoln, rbevins1@unl.edu

Follow this and additional works at: https://digitalcommons.unl.edu/psychfacpub

Part of the Psychiatry and Psychology Commons

Wilkinson, Jamie L.; Herrman, Laura; Palmatier, Matthew I.; and Bevins, Rick A., "Rats' novel object interaction as a measure of environmental familiarity" (2006). Faculty Publications, Department of Psychology. 41.

https://digitalcommons.unl.edu/psychfacpub/41

This Article is brought to you for free and open access by the Psychology, Department of at DigitalCommons@University of Nebraska - Lincoln. It has been accepted for inclusion in Faculty Publications, Department of Psychology by an authorized administrator of DigitalCommons@University of Nebraska - Lincoln. 


\title{
Rats' novel object interaction as a measure of environmental familiarity
}

\author{
Jamie L. Wilkinson, Laura Herrman, Matthew I. Palmatier, \\ and Rick A. Bevins \\ Department of Psychology, University of Nebraska-Lincoln, Lincoln, NE 68588-0308, USA \\ Submitted January 2005; revised April 2005; \\ published online June 2005; published May 2006.
}

\begin{abstract}
Environmental familiarization is a learning phenomenon embedded within most tasks used to study learning and motivation. Given its prevalence there is surprisingly little systematic behavioral research on factors affecting familiarization. The six experiments reported in the present report used rats' tendency to interact more with a novel object in a familiar than in a novel environment as a measure of environmental familiarization. We found that $3 \mathrm{~min}$ of exposure to the environment was sufficient to increase object interaction above unfamiliar controls even when testing occurred up to $48 \mathrm{~h}$ after initial exposure to the environment; 1 or $1.5 \mathrm{~min}$ of exposure was not sufficient. Also, in the brief 2 min test, 10 min of environment exposure did not appear to increase object interaction above the 3-min condition. The 3-min of environment exposure was sufficient for familiarization whether environment exposure occurred in one 3 min placement or two 1.5 min placements. Environmental familiarization as measured by object interaction was also sensitive to 'interference' manipulations. That is, a distinct object present during initial exposure to the environment produced a level of object interaction in testing comparable to an unfamiliar control. Similarly, exposure to a second distinct alternate environment immediately after, but not before, initial exposure to the test environment partially disrupted environmental familiarization. In sum, object interaction might serve as a useful measure for studying processes mediating environmental familiarity.

Keywords: Context learning, Curiosity, Exploratory behavior, Habituation, Hippocampus, Object recognition
\end{abstract}

Published in Learning and Motivation 37 (2006), pp. 131-148. Available online at www.sciencedirect.com DOI:10.1016/J.LMOt.2005.04.001 COPYRIGHT C 2005 ELSEVIER Inc. UsEd By PERMISSION. 
Typically, standard laboratory tasks used to study learning processes require that the animal be placed into an unfamiliar environment. Some experimental protocols include an explicit habituation or familiarization phase that precedes conditioning. For example, in a place conditioning experiment, many laboratories include one or 2 days of apparatus exposure in which the animal (e.g., rat) is allowed to freely explore the distinct environments that will later be paired with an aversive or appetitive stimulus [for a review, see Bardo and Bevins (2000)]. In contrast, other conditioning protocols simply start training with the implicit or explicit assumption that the animal will become familiar with the experimental environment and that this familiarization process will not significantly impact the learning process(es) of interest. Although this assumption is likely true for a great number of experiments, there are several published examples of differences in environmental familiarization affecting learning (e.g., Besheer and Bevins, 2000, Harrison and Isaac, 1984, Rowe et al., 1998, Williams et al., 1972 and Wilson et al., 2004). In the 1950s and 60s, there was a surge of behavioral research on environmental familiarization and associated processes. Much of this research was interested in distinguishing among concepts such as exploratory drive, instinct for curiosity, or emotionality to explain behavior change to novelty [for a more detailed discussion, see Fowler, 1965 and Hughes, 1997]. More recent research has focused on the physiological and pharmacological factors affecting environmental familiarization. The lack of comparable systematic behavioral research is surprising in that familiarization is a wide spread learning phenomenon that is embedded within most laboratory tasks used to study learning processes.

If one surveys the behavioral and the more extensive and recent physiological research that has been conducted on environmental familiarization, it becomes very clear that there is no standardized measure of familiarization. For example, many laboratories use a general decrease in activity as a measure of familiarization (e.g., Aloisi et al., 1997, Glickman and Hartz, 1964, Mason et al., 1998 and Montgomery, 1953). However, others suggest that rats' general activity measures more than familiarization and/or that changes in other behaviors such as sniffing, rearing, grooming, or turning might serve as a better measure of environmental familiarization (e.g., Brudzynski and Krol, 1997, Hughes, 1997, McCall et al., 1969 and Welker, 1959). There are likely many reasons for these differences in research outcome and opinion-non-standardized protocols, differences in apparatus, theoretical perspective, etc. Regardless, these differences have prompted some researchers to devise creative alternatives to line crossing or sniffing as indices of environmental familiarization. For example, Williams and Kuchta (1957) placed rats in an unfamiliar Y-maze in which each arm was distinctive. Located in one arm were 13 small objects fastened to the floor and side walls. On the first day of exposure rats spent a similar amount of time in each arm. Interestingly, on the last 4 days of exposure to the apparatus (days 5-8), the rats spent more time in the arm containing the objects than in the other two empty arms. This result suggested that object interaction might serve as a measure of environmental familiarization. Later, Sheldon (1969) confirmed this suggestion in a simplified version 
of this task. In that study, she repeatedly exposed rats to an elevated Y runway. At the end of the runway, was a novel object on one side and a familiar object on the other side of the Y. Across repeated exposures to the runway, rats shifted preference from the familiar object to the novel object suggesting that novel object interaction could serve as an index of familiarity with an environment.

More recently, we have taken advantage of this tendency for rats to interact more with a novel object when an environment is familiar to determine whether nicotine altered environmental familiarization (Bevins, Koznarova, \& Armiger, 2001). The two groups of interest from the perspective of the present report were the familiar group that received 4 min of exposure to the environment and the unfamiliar control that was equally exposed to handling and transport cues, but never experienced the environment before testing. On the test day, rats from both groups were placed in the environment for $2 \mathrm{~min}$; located on the opposite end of the placement area was a novel object. Rats in the familiar group interacted more with the object than unfamiliar controls. If rats were nicotine naïve, then pretreatment with $0.6 \mathrm{mg} / \mathrm{kg}$ of nicotine (salt form) interfered with environmental familiarization; object interaction was statistically similar to unfamiliar controls. Chronic pre-exposure to the same nicotine dose did not alter familiarization. We have also used this protocol to establish that withdrawal from nicotine (ca. 9-10 mg/kg/day for 7 days via osmotic pump) does not affect environmental familiarization (Besheer \& Bevins, 2003). Except for the acute effects of a moderate dose of nicotine that produces some impairment of activity, and hence less exploration, little is known about factors that affect this index of environmental familiarization. Indeed, its utility as an index of environmental familiarization will be linked to its sensitivity to experimental manipulations. Accordingly, the main goal of the present research was to systematically investigate variables that might affect environmental familiarization using novel object interaction as the primary dependent measure.

\section{Experiment 1}

Presumably there is a minimal amount of environment exposure time required for the rats to become familiar with an environment. To test this assumption, Experiment 1 assessed whether 1, 3, or $10 \mathrm{~min}$ of exposure to the environment was sufficient for familiarization if the test occurred $1 \mathrm{~h}$ after exposure.

\section{Methods}

\section{Animals}

The subjects were 32 naïve male Sprague-Dawley rats from Harlan (Indianapolis, IN) weighing $381 \pm 5 \mathrm{~g}$ at the start of the experiment. Rats were housed individually in plastic tubs lined with wood shavings. Food was continuously available in a temperature- and humidity-controlled colony set on a $12 \mathrm{~h}$ light/dark cycle. All exper- 
iments were conducted during the light cycle. Each rat was handled for at least $2 \mathrm{~min}$ daily for 3 days before the start of the experiment. The experimental protocols used in this report were approved by the University of Nebraska Institutional Animal Care and Use Committee and were conducted in accordance with the "Principles of Laboratory Animal Care" (NIH Publication No. 85-23, revised 1985).

\section{Apparatus}

A rectangular three section wooden box was used as the to-be-familiarized and the test chamber. Each end section was $31 \times 24 \times 45.5(1 \times \mathrm{w} \times \mathrm{h}) \mathrm{cm}$ with painted white walls, wire rod floors, and newspaper lining the litter tray. The center section was 15 $\times 24 \times 45.5(1 \times \mathrm{w} \times \mathrm{h}) \mathrm{cm}$ with a solid aluminum floor and gray walls. An $80 \mathrm{~dB}$ continuous white noise masked external sounds and a florescent ceiling light provided general illumination. The novel object was a peach colored paint roller $(7.5 \mathrm{~cm}$ long, $4 \mathrm{~cm}$ in diameter) attached to a plastic red scouring pad ( $9 \mathrm{~cm}$ diameter). All test sessions were videotaped for later observation (see Behavioral measures section).

\section{Procedure}

Rats were randomly assigned to one of four groups ( $\mathrm{n}=8$ per group). Rats assigned to the three exposed groups $(1,3$, or $10 \mathrm{~min})$ were placed against the wall at one end of the test environment and allowed free access to the environment for the number of minutes designated by their group's name before being returned to the home cage. Placement position was counterbalanced such that half of the rats in each group were placed sideways against the far wall of one end compartment. The fourth group of rats ( 0 min or Unfamiliar) was handled and transported to the experimental room in the same manner as the exposed groups, but they were not placed in the test environment. A 2-min test was conducted $1 \mathrm{~h}$ after environmental familiarization. Rats were placed against the wall at one end of the chamber and allowed unrestricted access to the environment for $2 \mathrm{~min}$. Located against the opposite end wall was the novel object. Placement was counterbalanced such that half the rats were placed in the same end as in the familiarization phase; the remaining rats were placed on the opposite side. The Unfamiliar group was not exposed to the chamber until the test session and thus provided a benchmark for the level of novel-object interaction when the test environment was completely novel (unfamiliar). Before the start of the experiment, the chamber and litter tray were cleaned. Also, between each day within the experiment, a "smeller rat" not included in the experiment was allowed to explore the test environment for $10 \mathrm{~min}$. This protocol was adopted because preliminary research (see also Bevins et al., 2001) indicated a high degree of variability in the behavior of the first rat regardless of group assignment. The "smeller rat" insures that an experimental rat is not the first to experience the environment and that the test chamber has rat odors distributed throughout.

\section{Behavioral measures}

For the test sessions, an experimenter naïve to group assignments scored the laten- 
cy to contact the novel object and the time spent interacting with the object in the test for all rats. Object interaction was defined as the time spent making 'directed' contacts with the object. Directed contacts include using the front paws or nose. Non-directed contacts were not counted; such behaviors include backing into the object or brushing against the object with the side or tail (cf. Besheer \& Bevins, 2003). Latency was scored as the time from placement until the rat made its first directed contact. To assess reliability of the primary rater, a second individual also naïve to group assignment scored these measures for two rats from each group in all six experiments. The Pearson's product moment correlation between the two observers on 44 rats was high for both measures: latency $(r=.99, p<.001)$, interaction $(r=.94, p<.001)$.

\section{Data analysis}

Object interaction and latency were analyzed with separate one-way analyses of variance (ANOVA). A significant $F$ value ( $\mathrm{p} \leq .05$, two-tailed rejection region) was followed up by Dunnett's multiple-comparison tests in which each group was compared to the Unfamiliar (0 min) control group.

\section{Results and discussion}

Environmental familiarization as indexed by object interaction varied as a function of exposure time, $\mathrm{F}(3,28)=12.74, \mathrm{p}<.001$ (see Fig. 1A). Dunnett's tests revealed that rats that spent 3 or $10 \mathrm{~min}$ in the environment during the familiarization phase interacted more with the object during testing than the unfamiliar control group ( $0 \mathrm{~min})$, ps $<.05$. A similar pattern was seen for latency to contact the object, $F(3,28)=4.31$, $\mathrm{p}=.013$ (see Fig. 1B). Rats that received 3 or $10 \mathrm{~min}$ of familiarization time made the initial contact with the object faster than unfamiliar controls, $\mathrm{ps}<.05$.

\section{Experiment 2}

Three minutes was sufficient for environmental familiarization when the test was conducted $1 \mathrm{~h}$ after exposure to the test apparatus. Experiment 2 extended this observation by determining whether 1,3 , or $10 \mathrm{~min}$ of exposure to the environment was sufficient if the test occurred $24 \mathrm{~h}$ after exposure.

\section{Method}

The subjects were 32 naïve male Sprague-Dawley rats (421 $\pm 10 \mathrm{~g})$. Housing, care, and apparatus were unchanged. Rats were randomly assigned to one of four groups: 0 min (Unfamiliar), 1, 3, or 10 min ( $\mathrm{n}=8$ per group). Procedures were identical to Experiment 1 except the test session was conducted $24 \mathrm{~h}$ after initial exposure.

\section{Results and discussion}

The one-way ANOVA for object interaction was significant, F $(3,28)=8.14$, p < .001 . Rats that were familiarized with the environment for 3 or 10 min interacted 

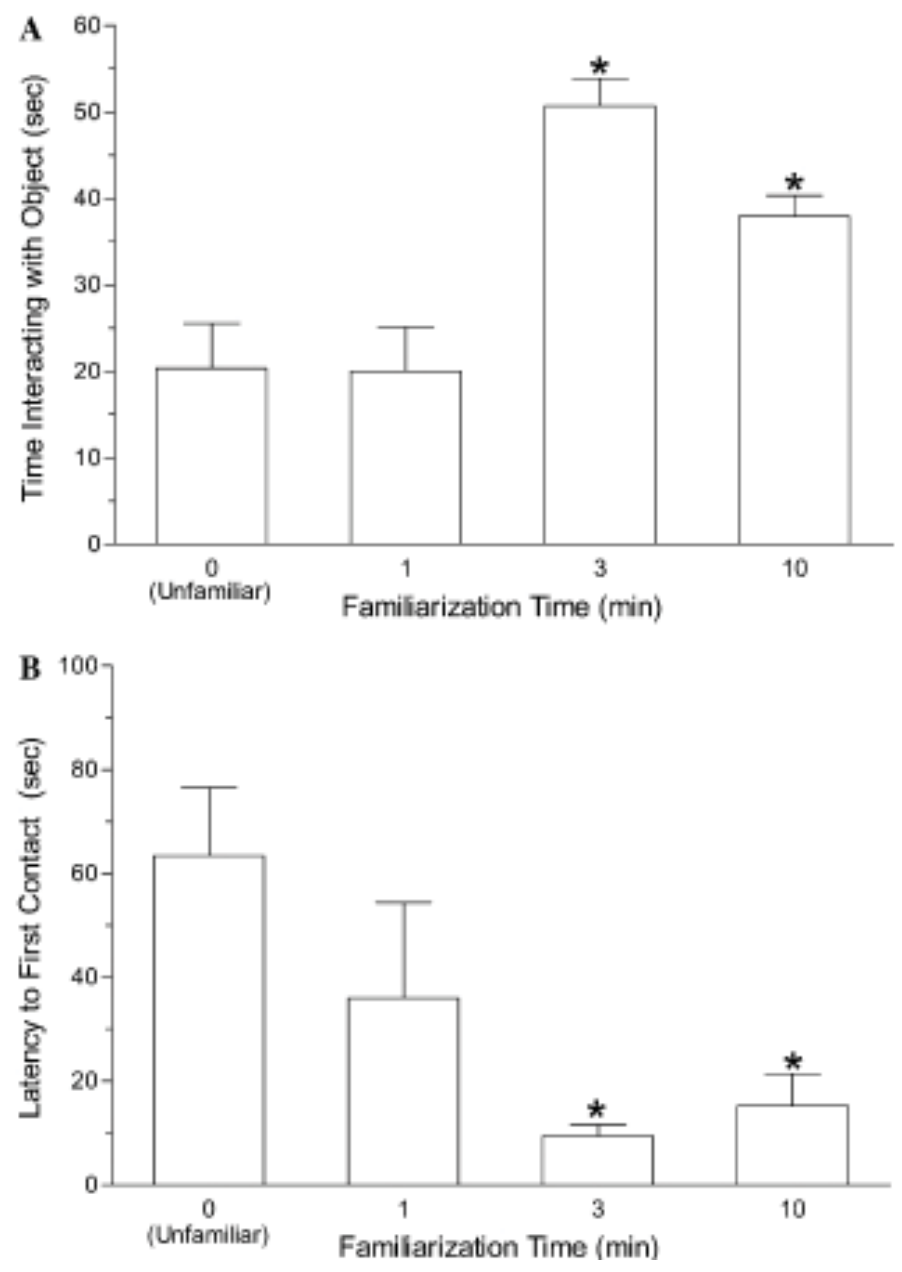

Fig. 1. (A) The mean time in seconds (+1 SEM) interacting with the novel object during the 2-min test of Experiment 1. (B) Time in seconds to Wrst contact the object during the test. * Significant difference $(\mathrm{p} \leq .05)$ from the Unfamiliar (0 $\mathrm{min}$ ) control group.

more with the novel object than the unfamiliar controls, ps $<.05$ (Fig. 2A). Latency to make first contact with the object showed a similar pattern (Fig. 2B); the one-way ANOVA was significant, $\mathrm{F}(3,28)=8.18, \mathrm{p}<.001$, with faster latencies relative to the unfamiliar controls occurring after 3 and $10 \mathrm{~min}$ of exposure, ps $<.05$.

\section{Experiment 3}

Experiments 1 and 2 established that 3 min of environment exposure was sufficient for familiarization up to $24 \mathrm{~h}$ later if exposure occurred in the same placement. Experiment 3 sought to extend this observation by determining whether 3 min of exposure was sufficient if that exposure occurred on two separate occasions (1.5 min each) sep- 

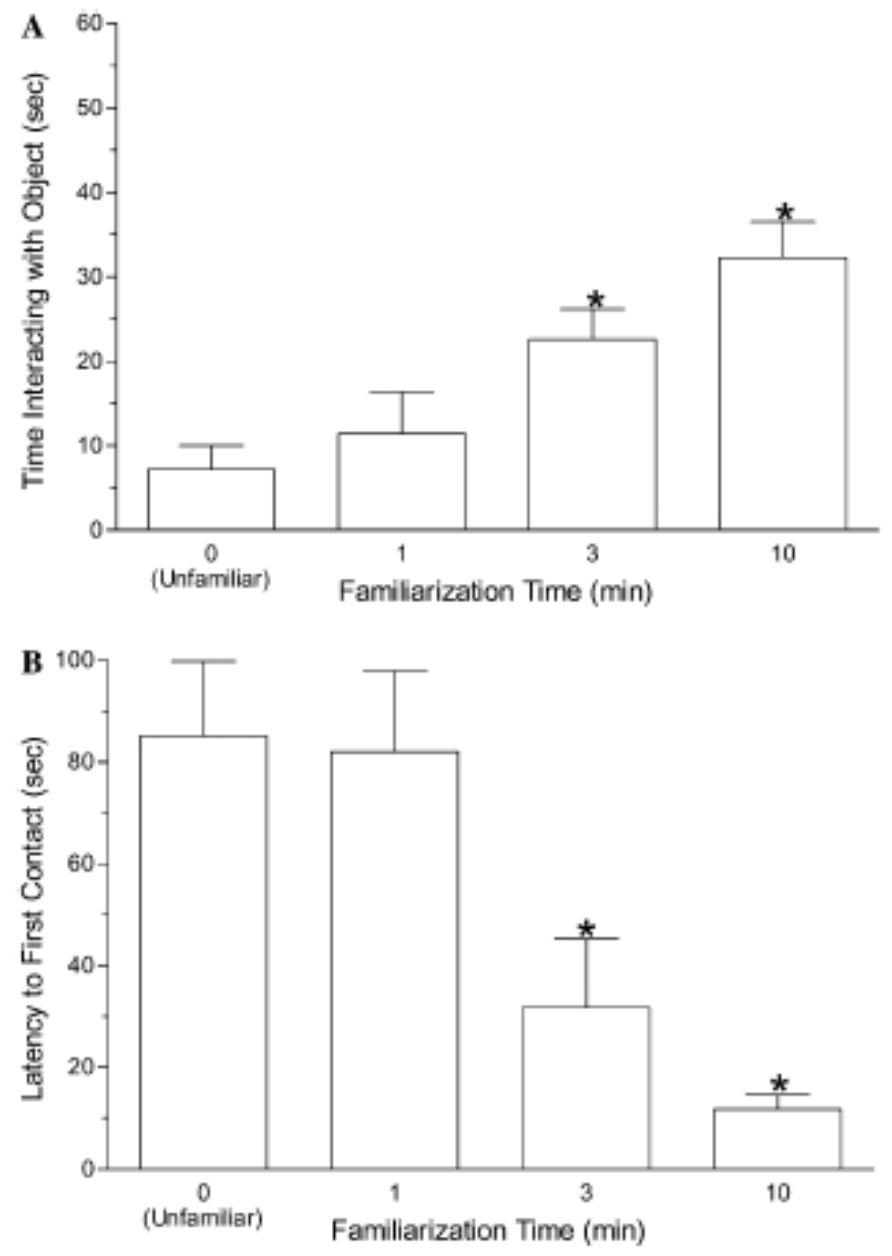

Fig. 2. (A) The mean time in seconds (+1 SEM) interacting with the novel object during the 2-min test of Experiment 2. (B) Time in seconds to first contact the object during the test. *Significant difference $(p \leq .05)$ from the Unfamiliar ( $0 \mathrm{~min}$ ) control group.

arated by $24 \mathrm{~h}$. Experiments 1 and 2 also established that 1 min was not sufficient to significantly increase object interaction (i.e., rats not familiar). One of the comparison conditions of Experiment 3 examined whether $30 \mathrm{~s}$ more $(1.5 \mathrm{~min})$ was sufficient for environmental familiarization.

\section{Method}

Apparatus and care of the rats was unchanged. Experimentally naïve rats $(261 \pm 2$ g) were randomly assigned to one of four groups: $0 \mathrm{~min}$ (Unfamiliar), $1.5 \mathrm{~min}, 1.5(2)$, or $3 \min (n=10$ per group). Days 1 and 2 were the familiarization phase. On Day 1, rats in group 1.5(2) were placed in the chamber for $1.5 \mathrm{~min}$ as described earlier. Half 
the rats in group 1.5 and 3 min were placed in the chamber for the assigned time. All other rats including the unfamiliar controls received the same handling and transport experience, but were not placed in the chamber. On Day 2, all rats in group 1.5(2) were again placed in the chamber for $1.5 \mathrm{~min}$. Thus, these rats received a total of 3 min exposure to the chamber, but that exposure was split into two equal durations (1.5 min) each separated by $24 \mathrm{~h}$. Rats in the 1.5 and 3 min groups that were not exposed to the environment on Day 1 were placed in the chamber for the assigned time. Rats not scheduled to have environment exposure on Day 2 received comparable handling and transport experience. On Day 3, the 2 min novel-object test was conducted as described earlier.

\section{Results and discussion}

Note that group 1.5 and $3 \mathrm{~min}$ had a subset of rats that received environmental familiarization $48 \mathrm{~h}$ before the test; the other subset was familiarized $24 \mathrm{~h}$ before testing. The subsets for each group did not differ statistically on latency to contact or object interaction and were thus combined for graphic display and subsequent analyses. There was evidence for environmental familiarization as indexed by object interaction whether exposure occurred in one 3 min placement or in two 1.5 min placements (see Fig. 3A). The one-way ANOVA was significant, $\mathrm{F}(3,36)=4.40, \mathrm{p}=.0098$. Rats in groups 1.5(2) and $3 \mathrm{~min}$ interacted more with the novel object on the test day than the unfamiliar $(0 \mathrm{~min})$ control group, $\mathrm{ps}<.05 ; 1.5 \mathrm{~min}$ of exposure before the test was not sufficient to significantly increase object interaction. In contrast to previous experiments, latency to first contact the object did not parallel object interaction data. That is, the overall ANOVA was not significant, $\mathrm{F}<1$, indicating that latency did not vary as a function of exposure time (see Fig. 3B). Indeed, relative to the earlier experiments, the unfamiliar control latencies appear lower. One notable difference was that all rats in the present experiment were handled and transported to the test room twice; rats in Experiments 1 and 2 only received one exposure to these handling/transport cues. Perhaps, latency to first contact is more sensitive than overall object interaction to experience with handling and transport to the experimental room.

\section{Experiment 4}

An explicit assumption underlying the object interaction measure of environmental familiarization is that in the unfamiliar controls the behaviors evoked by the novelty of the environment interfere with object interaction (cf. Bevins et al., 2001). If so, the reverse should be true. That is, if a novel object was present during the familiarization phase, interaction with that object should compete with the behaviors required for environmental familiarization. Experiment 4 tested this prediction by comparing the standard familiar and unfamiliar controls with a group that had an object present during the familiarization phase; that object differed from the one used during testing. 

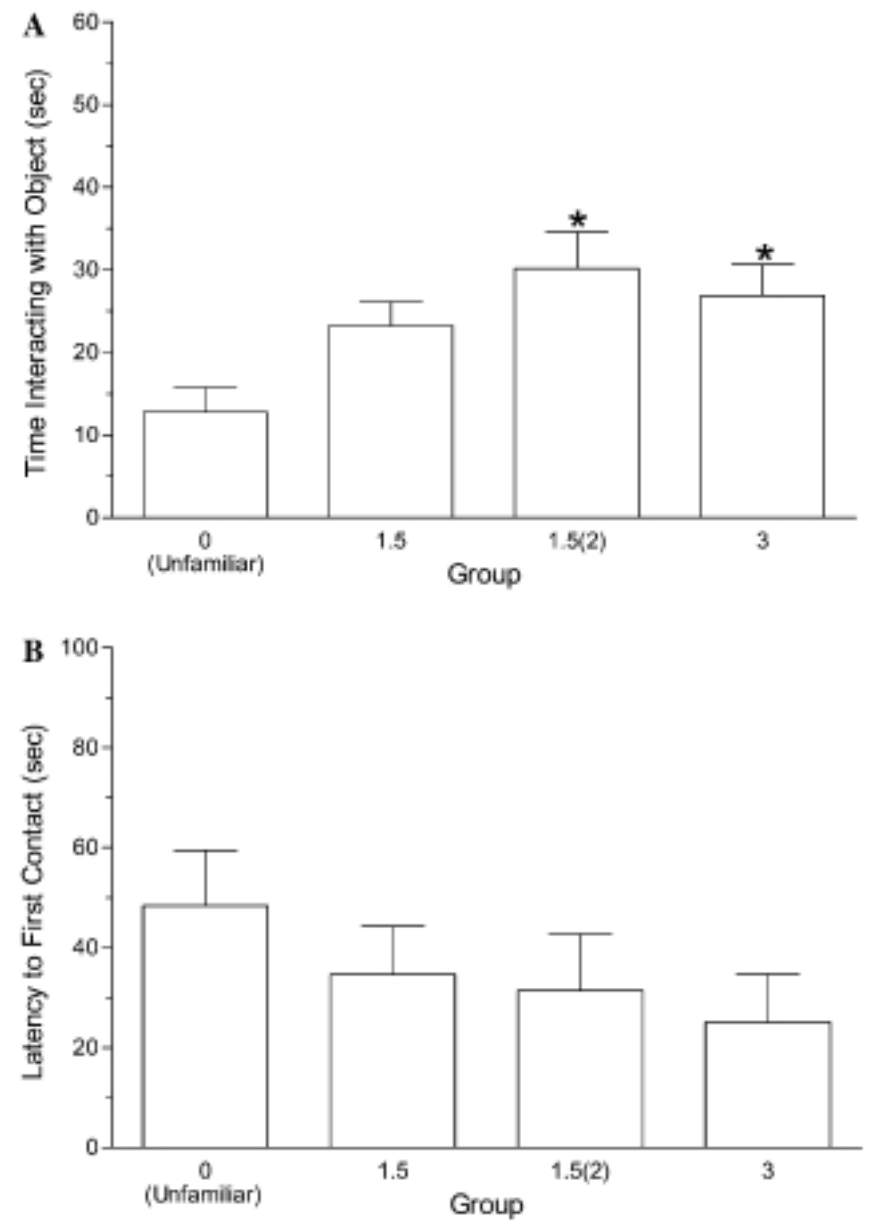

Fig. 3. (A) The mean time in seconds (+1 SEM) interacting with the novel object during the 2-min test of Experiment 3. (B) Time in seconds to first contact the object during the test. *Significant difference $(\mathrm{p} \leq .05)$ from the Unfamiliar control group.

\section{Method}

\section{Animals and apparatus}

Care and housing of the rats $(267 \pm 2 \mathrm{~g})$ was unchanged. Besides the addition of a second object, a white loofa (diameter approximately $12 \mathrm{~cm}$ ), the materials and apparatus were similar to the previous experiments. Previous research in our laboratory indicates that the loofa is discriminable from the roller-pad and maintains comparable levels of object interaction (Besheer, Jensen, \& Bevins, 1999).

\section{Procedures}

Rats were randomly assigned to one of three groups: Unfamiliar, Familiar (No Object), or Familiar (Object) $(n=8$ per group). On Day 1, rats in the familiar groups 
were placed in the chamber for $3 \mathrm{~min}$ as described in Experiment 1. However, for rats in the Familiar (Object) an object (roller-pad or loofa) was located in the center compartment of the chamber. Rats in the Unfamiliar group were handled and transported in a manner similar to the other groups. To control for the effects of mere object exposure, rats in the Unfamiliar and Familiar (No Object) groups had 3 min of access to an object in the home cage $24 \mathrm{~h}$ after environment exposure. Half of the rats in each group were exposed to the loofa during the familiarization phase; the remaining rats receive exposure to the roller pad. The test for environmental familiarization was conducted $48 \mathrm{~h}$ after the environment exposure. Rats were tested with the object not used in familiarization phase (i.e., object was novel). All other testing procedures were identical to previous experiments.

\section{Results and discussion}

For each group, which object served as the novel object during testing did not significantly affect the interaction or latency measure. Accordingly, data were pooled across object-exposure conditions for subsequent analyses and graphic display. An object present during the familiarization phase interfered with environmental familiarization as measured by novel object interaction (Fig. 4A). For object interaction, there was a significant one-way ANOVA, $F(2,21)=4.11, p=.031$. Only rats that were familiarized without an object present interacted more with the novel object on the test day, $\mathrm{p}<.05$. As in Experiment 3, object interaction appeared to be the more sensitive measure of environmental familiarization (Fig. 4B). Average contact latency was relatively quick and statistically similar across groups, $\mathrm{F}(2,21)=1.15, \mathrm{p}=0.337$.

A novel object present during initial exposure to an environment interfered with familiarization as measured by object interaction in the 2-min test. A competing behavior account of this result argues that a rat cannot explore the environment if it is exploring the object (i.e., behaviors are incompatible). An alternative to this competing behavior account suggests that the object was an important feature of the environment. Thus, when it was switched with a different object during testing the environment regained some novelty. Regardless, the object present during the familiarization phase altered subsequent familiarity with the environment as measured by interaction with a novel object. Notably, mere experience with an object cannot account for group differences. The unfamiliar and familiar controls received similar access to the object in the home cage $24 \mathrm{~h}$ after initial exposure to the environment. This object exposure, however, might be responsible for the relatively quick latency to make first contact with the object during testing in all groups. This dissociation between latency and object interaction (see also Experiment 3 ) suggests that the measures are sensitive to different functional relations. For example, latency appears to be more sensitive than object interaction to 'external' environment experience such as handling, transport, and object exposure. This suggestion will require explicit testing. Finally, to allow for object exposure in the home cage, the placement to testing interval was extended to 48 

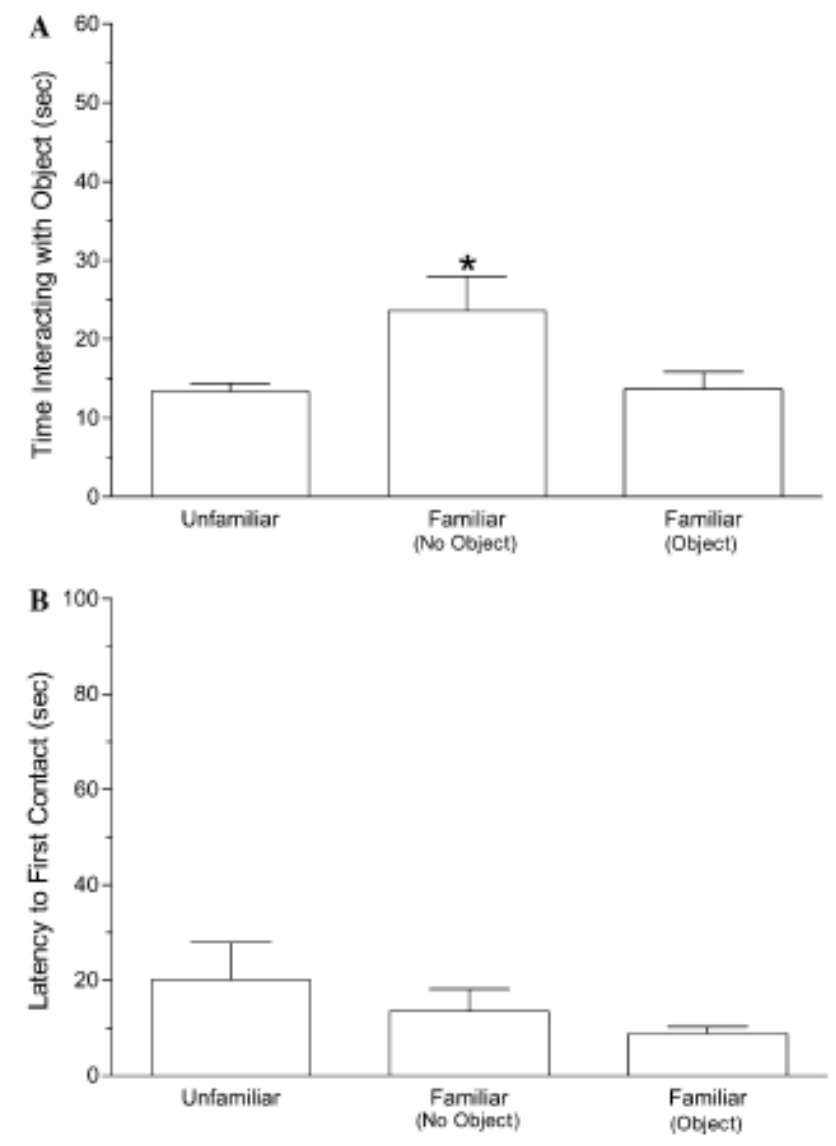

Fig. 4. (A) The mean time in seconds (+1 SEM) interacting with the novel object during the 2-min test of Experiment 4. (B) Time in seconds to first contact the object during the test. *Significant difference $(\mathrm{p} \leq .05)$ from the Unfamiliar control group.

h. The difference between group Unfamiliar and Familiar (No Object) indicates that 3 min of environment exposure is retained for $48 \mathrm{~h}$. This observation replicates results from group 3 min of Experiment 3. In that experiment, the subset of rats that received a 48-h retention interval did not differ statistically from the subset that received a 24$\mathrm{h}$ retention interval.

\section{Experiment 5}

Experiment 4 established that simultaneous presentation of two different stimuli (environment versus object) interfered with subsequent recall of the environment. A natural extension of this finding would be to determine if sequential presentation of two different stimuli affected familiarization. To do so, in Experiment 5 we exposed rats to a second distinct environment immediately after initial exposure to the test environment. 


\section{Method}

\section{Animals and apparatus}

Housing and care of the rats was unchanged. The design of Experiment 5 required a second environment that was distinctly different from the rectangular white chamber that served as the test environment in Experiments $1-4$. The alternate environment was a black metal mailbox measuring $48 \times 16.5 \times 22(1 \times \mathrm{w} \times \mathrm{h}) \mathrm{cm}$. The mailbox top had 15 holes $(1 \mathrm{~cm}$ each) that allowed airflow when the door was closed.

\section{Procedure}

Rats $(293 \pm 5 \mathrm{~g})$ were randomly assigned to one of four groups: T0/A0, T0/A3, T3/ $\mathrm{A} 0$, or $\mathrm{T} 3 / \mathrm{A} 3$ ( $\mathrm{n}=12$ per group). The letters denote the apparatus $[\mathrm{T}=$ test chamber (white rectangular box); $\mathrm{A}=$ alternate environment (mailbox)]. Number after the letter indicates the time spent in that environment. Thus, group T3/A0 is our standard familiar group in which rats received $3 \mathrm{~min}$ of exposure to the white rectangular box and no exposure to the alternate environment. The Unfamiliar group is T0/A0. Group T0/A3 received only $3 \mathrm{~min}$ of exposure to the alternate environment and served to determine whether exposure to any environment might increase object interaction similar to that of a familiar group. Finally, group T3/A3 received exposure to both environments. Rats in this group were placed in the test chamber for $3 \mathrm{~min}$ as described earlier and then immediately placed in the alternate environment for $3 \mathrm{~min}$ before being returned to the colony. Similar to previous experiments, handling and transport was equated across groups; only environment exposure was allowed to vary. The test session was $48 \mathrm{~h}$ after the familiarization phase and was conducted as described in Experiment 1.

Results and discussion

There was a tendency for exposure to the alternative environment to disrupt familiarization as measured by object interaction (Fig. 5A). The one-way ANOVA was significant, $\mathrm{F}(3,44)=4.0, \mathrm{p}=.013$. Subsequent Dunnett's tests revealed that only group T3/A0 (Familiar) interacted significantly more with the novel object than unfamiliar control (T0/A0), $\mathrm{p}<.01$. The ANOVA for latency was also significant, $\mathrm{F}(3,44)=$ $3.86, \mathrm{p}=.016$. Rats exposed to the test environment (i.e., groups $\mathrm{T} 3 / \mathrm{A} 0$ and $\mathrm{T} 3 / \mathrm{A} 3$ ) had faster latencies than the unfamiliar control group, ps $<.05$ (Fig. 5B).

Similar to previous experiments, there appears to be a dissociation between the latency measure and the object interaction measure. Rats that received exposure to the alternative environment immediately following familiarization to the test environment (group T3/A3) had a faster latency to contact the novel object on the test day, but object interaction in the 2-min test did not differ significantly from the unfamiliar control. Although the object interaction data might be taken as retroactive interference (cf. Escobar and Miller, 2003, Izquierdo and Pereira, 1989, Slamecka and Ceraso, 1960 and Terry, 1996), in a post hoc comparison, group T3/A3 had an intermediate level of object interaction that did not differ significantly from group Familiar, $t(22)=1.76$, 

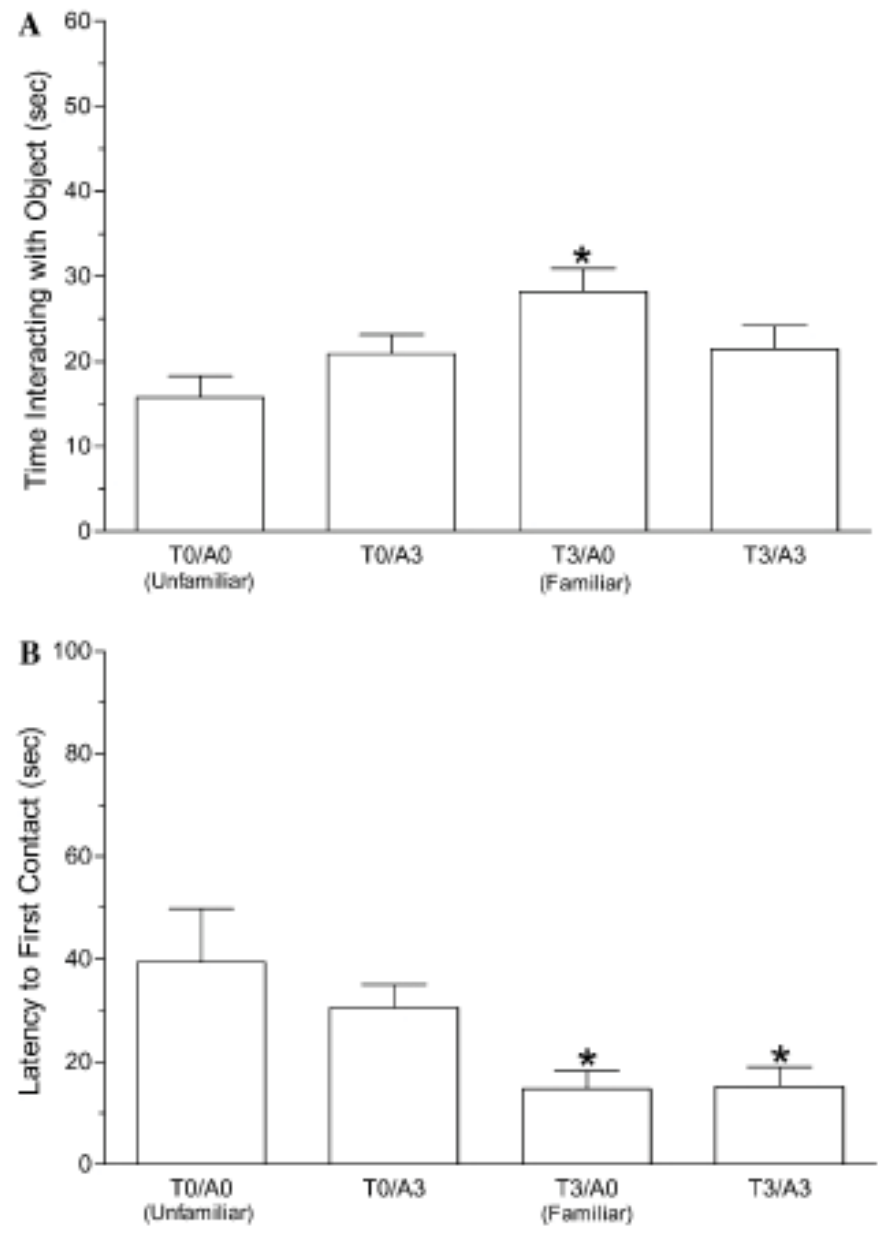

Fig. 5. (A) The mean time in seconds (+1 SEM) interacting with the novel object during the 2-min test of Experiment 5. (B) Time in seconds to first contact the object during the test. * Significant difference $(p \leq .05)$ from the Unfamiliar (T0/A0) control group.

$\mathrm{p}=.092$. The finding that group T3/A3 did not differ from the Familiar or Unfamiliar group suggests that familiarity was, at best, only partially disrupted by alternative environment exposure. Finally, relative to unfamiliar controls, $3 \mathrm{~min}$ in the alternative environment did not affect latency or object interaction. This finding is important because it suggests that familiarization - increased object interaction-required more than exposure to any environment for $3 \mathrm{~min}$. That is, exposure to the actual test environment (or one that share stimulus properties) seems to be required.

\section{Experiment 6}

Experiment 6 sought to determine whether the pattern of disruption would be more or less pronounced if order of environment exposure was reversed. That is, would ex- 
posure to the distinct and alternate environment immediately before familiarization to the test environment alter object interaction (i.e., familiarization)?

\section{Method}

Housing, care, and apparatus were unchanged from Experiment 5. Rats (295 \pm 2 g) were randomly assigned to one of 3 groups: A0/T0 (Unfamiliar), A0/T3 (Familiar), or A3/T3 ( $n=8$ per group). The procedures were identical to Experiment 5 except rats in group A3/T3 were placed in the alternate environment before placement in the test chamber.

\section{Results and discussion}

Exposure to the alternative environment before placement in the test environment did not disrupt environmental familiarization (Fig. 6A). The ANOVA on object interaction was significant, $F(2,21)=6.76, p=.005$. Rats exposed to the test environment (group A0/T3 and A3/T3) interacted more with the novel object than the unfamiliar control rats, $\mathrm{ps}<.05$. Although the mean latency to contact the object was higher in the Unfamiliar controls, this tendency was not significant, $F(2,21)=1.92, p=0.172$.

\section{General discussion}

Familiarization with contextual stimuli occurs in most experimental protocols designed to elucidate learning and motivation processes. Whether this prevalent form of learning is treated explicitly in an experiment with a habituation phase, or is merely ignored, varies widely in the literature. Although environmental familiarization in many conditioning situations likely has little to no effect, there are published examples of differences in familiarization affecting learning (Besheer and Bevins, 2000, Harrison and Isaac, 1984, Rowe et al., 1998, Williams et al., 1972 and Wilson et al., 2004). Given the prevalence of environmental familiarization as a learning process, and its potential influence on acquisition and/or performance in some learning experiments, there is surprisingly little systematic behavioral research on factors affecting familiarization. To begin to address this deficit, the present research used rats' tendency to interact more with a novel object in a familiar than in a novel environment as a measure of environmental familiarization (Besheer and Bevins, 2003, Bevins et al., 2001 and Sheldon, 1969). In brief, we found that $3 \mathrm{~min}$ of exposure to the environment was sufficient to increase object interaction above unfamiliar controls even when testing occurred up to $48 \mathrm{~h}$ after initial exposure to the environment; 1 or 1.5 min was not sufficient. Also, in the brief 2 min test, 10 min of environment exposure did not appear to significantly increase object interaction above the 3-min condition. Whether this lack of difference reflects a ceiling effect of the brief test, lack of sensitivity in the use of object interaction as a measure of familiarization, or an all-or-none 

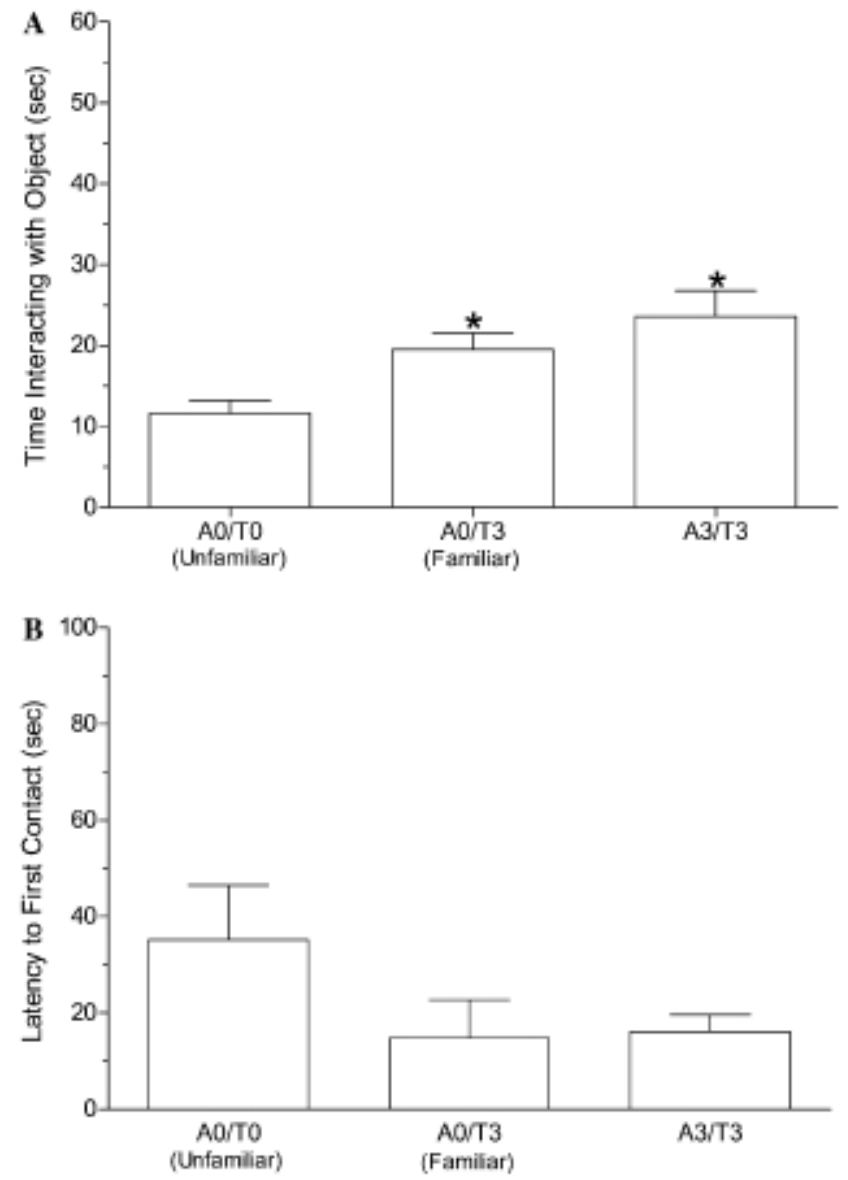

Fig. 6. (A) The mean time in seconds (+1 SEM) interacting with the novel object during the 2-min test of Experiment 6. (B) Time in seconds to first contact the object during the test. *Significant difference $(\mathrm{p} \leq .05)$ from the Unfamiliar (A0/T0) control group.

nature of familiarization in the present protocol will require further experiments. Notably, 3 min was sufficient for familiarization whether environment exposure occurred in one 3 min placement or two 1.5 min placements. Further, familiarization as measured by object interaction was sensitive to 'interference' manipulations. That is, an object present during initial exposure to the environment produced a level of object interaction comparable to an unfamiliar control. Exposure to a distinct alternate environment immediately after, but not before, initial exposure partially disrupted environmental familiarity.

The finding that an object located in the environment during initial exposure affected environmental familiarization parallels an earlier published observation from our laboratory. In that research, rats' ability to discriminate a novel versus familiar sample object (i.e., object recognition) was affected by familiarity with the environment 
on the day in which the sample object was first exposed (Besheer \& Bevins, 2000; see Experiment 1). That is, placement into a novel (unfamiliar) environment interfered with learning about the sample object such that rats could not later discriminate that sample object from a completely different and novel object. This discrimination failure was interpreted as a lack of familiarity with the sample object because of competing behaviors evoked by the environment. We suggest that a similar competing behavior account explains the lack of environmental familiarity when an object is present in the to-be-tested environment. That is, a rat cannot explore the environment if it is exploring the object (i.e., behaviors are incompatible). However, as noted earlier, an alternative to this competing behavior account suggests that the object was an important feature of the environment. Thus, when that object was replaced with a different object on the test day the environment regained its novelty. Unfortunately, because a novel environment interferes with object interaction (present experiments) and vice versa (Besheer \& Bevins, 2000, Experiment 1), distinguishing between these two accounts will not be easy. At present, we can only conclude that novelty (i.e., stimulus change) during the initial sampling or test phase interferes with familiarization.

The results described in the previous paragraph indicate that familiarization can be disrupted with a manipulation that occurs during initial environment exposure (i.e., presence of a novel object). Familiarization as measured by object interaction was not nearly as disrupted if rats were exposed to a second distinct environment immediately before or after initial environment exposure. This pattern of results suggests that exposure to additional stimuli (an object) during familiarization is more disruptive than presentation of a second environment immediately before or after familiarization. Exposure to the alternative environment appeared slightly more disruptive to familiarization if the exposure was after (group T3/A3 did not differ from Familiar or Unfamilar control) rather than before (group A3/T3 was significantly higher than Unfamiliar control) the familiarization phase. Although caution should be taken when comparing across experiments, future research systematically exploring whether alternative manipulations after familiarization could produce complete disruption might lead to a model useful for studying factors mediating retroactive interference of environmental familiarization (cf. Escobar and Miller, 2003, Izquierdo and Pereira, 1989, Slamecka and Ceraso, 1960 and Terry, 1996). Such manipulations might include longer exposure to the alternative environment, alterations in that environment's features, or the addition of a mild stressor.

We also measured latency to make first directed contact with the object in testing. When experiments involved a single manipulation 'external' to the familiarization experience (e.g., handling/transport in unfamiliar controls of Experiment 1) then the latency measure paralleled the interaction measure. That is, latency to contact was faster in groups that interacted more with the object than unfamiliar controls (see Experiments 1 and 2). However, this parallel fell apart when additional 'external' experiences were added to control for various experimental manipulations. For example, in Experiment 3 rats in the 'standard' unfamiliar and familiar conditions were handled and 
transported to the test room twice to match this experience with group 1.5(2); there were no differences among the groups in latency (see also Experiment 6). Further, in Experiment 4 unfamiliar and familiar controls received access to an object in the home cage $24 \mathrm{~h}$ after initial exposure to the environment to match object experience with the Familiar (Object) group. Again there were no differences in latency to contact the object. This dissociation between latency and object interaction suggests that the measures are sensitive to different functional relations. For example, latency appears to be more sensitive than object interaction to 'external' environment experience such as handling, transport, and object exposure. However, the experiments in the present report were not designed to test this possibility. Future studies testing this possibility will need to include appropriate and systematic comparisons for differential handling/transport experience that do not predict a null effect.

Regardless, the present experiments (see also Besheer and Bevins, 2003, Bevins et al., 2001, Sheldon, 1969 and Williams and Kuchta, 1957) provide a good initial demonstration of the potential utility of using novel object interaction as a measure of environmental familiarization. An appreciation of the functional relation between behavioral and neurobiological variables and environmental familiarization seems important given that it is a learning phenomenon that is embedded in most of the tasks used to study learning and motivation. For example, does familiarization progress similarly in different shape environments? How does environmental complexity affect familiarization? Will pharmacological manipulations shown to alter other memory processes affect familiarization? Does a simultaneously required learning task slow familiarization? Recent research by Wilson et al. (2004) suggests that age differences in spatial learning likely reflect differences in the way the hippocampus in old versus young rats represents new environments. That is, the neurobiological processes mediating environmental familiarization differ across age and these differences result in spatial learning deficits in old rats. This finding makes us wonder how many other differences in learning reflect differences in environmental familiarization.

\section{Acknowledgments}

Funds from USPHS Grants DA011893 and UNL Research Council supported the research; R. Bevins was partially supported by Grant DA018114 while writing this manuscript. We are grateful to Hannah Siebert for conducting interobserver reliability checks and Matthew Anderson and Jennifer Murray for their thoughtful comments on an earlier version of this manuscript. M. Palmatier is now in the Department of Neuroscience at the University of Pittsburgh.

\section{References}

Aloisi et al., 1997: A.M. Aloisi, F. Casamenti, C. Scali, G. Pepeu and G. Carli, Effects of novelty, pain and stress on hippocampal extracellular acetylcholine levels in male rats, Brain Research 748 (1997), pp. 219-226.

Bardo and Bevins, 2000: M.T. Bardo and R.A. Bevins, Conditioned place preference: What does it add to our preclinical understanding of drug reward?, Psychopharmacology 153 (2000), pp. 31-43. 
Besheer et al., 1999: J. Besheer, H.C. Jensen and R.A. Bevins, Dopamine antagonism in a novel-object preference and a novel-object place conditioning preparation with rats, Behavioural Brain Research 103 (1999), pp. 35-44.

Besheer and Bevins, 2000: J. Besheer and R.A. Bevins, The role of environmental familiarization in novelobject preference, Behavioural Processes 50 (2000), pp. 19-29.

Besheer and Bevins, 2003: J. Besheer and R.A. Bevins, The impact of nicotine withdrawal on novelty reward and related behaviors, Behavioral Neuroscience 117 (2003), pp. 327-340.

Bevins et al., 2001: R.A. Bevins, J. Koznarova and T.J. Armiger, Environmental familiarization in rats: Differential effects of acute and chronic nicotine, Neurobiology of Learning and Memory 75 (2001), pp. 63-76.

Brudzynski and Krol, 1997: S.M. Brudzynski and S. Krol, Analysis of locomotor activity in the rat: Parallelism index, a new measure of locomotor exploratory pattern, Physiology and Behavior 62 (1997), pp. 635-642.

Escobar and Miller, 2003: M. Escobar and R.R. Miller, Timing in retroactive interference, Learning and Behavior 31 (2003), pp. 257-272.

Fowler, 1965: H. Fowler, Curiosity and exploratory behavior, Macmillan, NY (1965).

Glickman and Hartz, 1964: S.E. Glickman and K.E. Hartz, Exploratory behavior in several species of rodents, Journal of Comparative and Physiological Psychology 58 (1964), pp. 101-104.

Harrison and Isaac, 1984: D.W. Harrison and W. Isaac, Disruption and habituation of stable fixed-interval behavior in younger and older monkeys, Physiology and Behavior 32 (1984), pp. 341-344.

Hughes, 1997: R.N. Hughes, Intrinsic exploration in animals: motives and measurement, Behavioural Processes 41 (1997), pp. 213-226.

Izquierdo and Pereira, 1989: I. Izquierdo and M.E. Pereira, Post-training memory facilitation blocks extinction but not retroactive interference, Behavioral and Neural Biology 51 (1989), pp. 108-113.

Mason et al., 1998: K. Mason, D.J. Heal and S.C. Stanford, The anxiogenic agents, yohimbine and FG 7142, disrupt the noradrenergic response to novelty, Pharmacology Biochemistry and Behavior 60 (1998), pp. 321-327.

McCall et al., 1969: R.B. McCall, M.L. Lester and C.G. Dolan, Differential rearing and the exploration of stimuli in the open field, Developmental Psychology 1 (1969), pp. 750-762.

Montgomery, 1953: K.C. Montgomery, Exploratory behavior as a function of "similarity" of stimulus situations, Journal of Comparative and Physiological Psychology 46 (1953), pp. 129-133.

Rowe et al., 1998: W.B. Rowe, E. Spreekmeester, M.J. Meaney, R. Quirion and J. Rochford, Reactivity to novelty in cognitively impaired and cognitively unimpaired aged rats and young rats, Neuroscience 83 (1998), pp. 669-680.

Sheldon, 1969: A.B. Sheldon, Preference for familiar versus novel stimuli as a function of the familiarity of the environment, Journal of Comparative and Physiological Psychology 67 (1969), pp. 516-521.

Slamecka and Ceraso, 1960: N.J. Slamecka and J. Ceraso, Retroactive and proactive inhibition of verbal learning, Psychological Bulletin 57 (1960), pp. 449-475.

Terry, 1996: W.S. Terry, Retroactive interference effects on surprising reward omission on serial spatial memory, Journal of Experimental Psychology: Animal Behavior Processes 22 (1996), pp. 472-479.

Welker, 1959: W.I. Welker, Escape, exploratory, and food seeking responses of rats in a novel situation, Journal of Comparative and Physiological Psychology 52 (1959), pp. 106-111.

Williams and Kuchta, 1957: C.D. Williams and J.C. Kuchta, Exploratory behavior in two mazes with dissimilar alternatives, Journal of Comparative and Physiological Psychology 50 (1957), pp. 509-513.

Williams et al., 1972: D.I. Williams, U. Hull and G. Lowe, Light reinforcement in the rat: The effects of continuous and discontinuous periods of apparatus familiarization, Quarterly Journal of Experimental Psychology 24 (1972), pp. 98-101.

Wilson et al., 2004: I.A. Wilson, S. Ikonen, I. Gureviciene, R.W. McMahan, M. Gallagher and H. Eichenbaum et al., Cognitive aging and the hippocampus: How old rats represent new environments, Journal of Neuroscience 24 (2004), pp. 3870-3878. 